\title{
High Content Phenotypic Screen of a Focused TCAMS Drug Library Identifies Novel Disruptors of the Malaria Parasite Calcium Dynamics
}

Wanni Chia, ${ }^{\text {a\# }}$ Maria G. Gomez-Lorenzo, ${ }^{\mathrm{b}}$ Isabel Castellote, ${ }^{\mathrm{b}}$ Jie Xin Tong, ${ }^{\text {a }}$ Rajesh Chandramohanadas, ${ }^{\mathrm{a}}$ Trang Thi Thu Chu, ${ }^{\mathrm{a}}$ Wanxiang Shen, ${ }^{\mathrm{c}}$ Mei Lin Go, ${ }^{\mathrm{c}}$ Cristina de Cozar, ${ }^{\mathrm{b}}$ Benigno Crespo, ${ }^{\mathrm{b}}$ Maria J. Almela,${ }^{\mathrm{b}}$ Fernando Neria-Serrano, ${ }^{\mathrm{b}}$ Virginia Franco, ${ }^{\mathrm{b}}$ FranciscoJavier Gamo, ${ }^{\mathrm{b}}$ Kevin S. W. Tan ${ }^{\mathrm{a} *}$

aLaboratory of Molecular and Cellular Parasitology, Department of Microbiology and Immunology, and Healthy Longevity Translational Research Programme, Yong Loo Lin School of Medicine, National University of Singapore, S117545, Singapore.

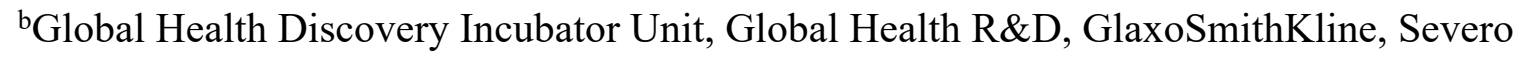
Ochoa 2, Tres Cantos 28760, Madrid Spain.

${ }^{c}$ Department of Pharmacy, National University of Singapore, 18 Science Drive 4, S117543, Singapore.

Running title: Novel Disruptors of Malaria Parasite Calcium Dynamics

*Address correspondence to: Kevin SW Tan, mictank@,nus.edu.sg

${ }^{\#}$ Current address: Program in Emerging Infectious Diseases, Duke-NUS Graduate Medical School, S169857, Singapore.

${ }^{\$}$ Current address: Procter \& Gamble Innovation Center, 70 Biopolis St, S138547, Singapore 


\section{Supplementary Figures and Tables.}

\section{Supplemental Data S1}

a.

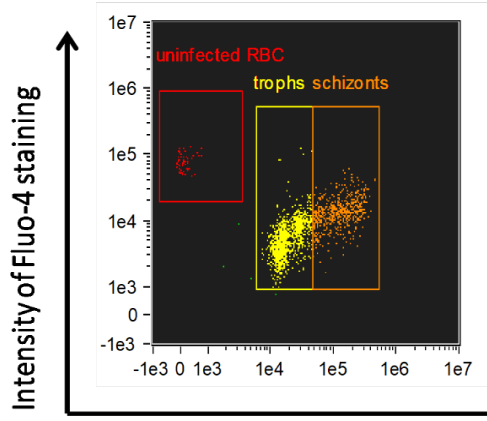

b.

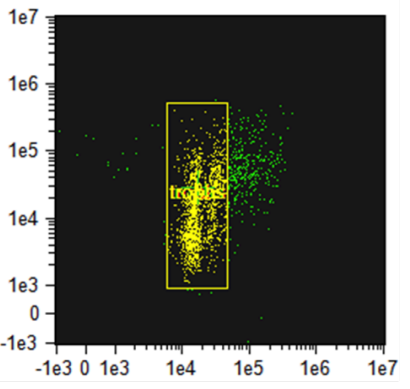

c.

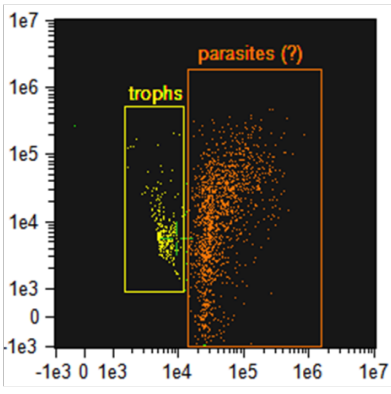

d.

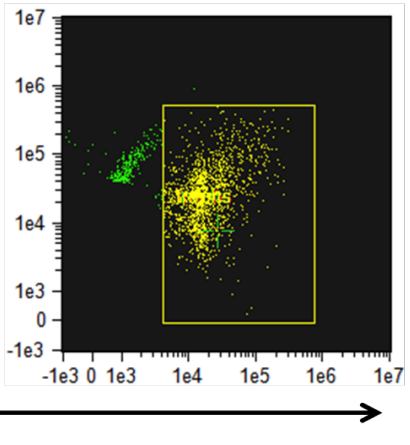

Intensity of Hoechst staining

10 compounds with morphological changes after drug treatment in AMNIS. a. Representative AMNIS plot of trophozoites with Fluo-4 localized in the parasite after PBS treatment. b. Representative AMNIS plot of trophozoites with relocalized calcium ions after CQ treatment. c. Representative AMNIS plot of trophozoites with big scatterplots compared to CQ treatment plots after drug treatment (Compounds: TCMDC-139960, -132968, -136977, -133201, -139622, -138938 and -132920). d. Representative AMNIS plot of trophozoites with small scatterplots compared to chloroquine after drug treatment (Compounds: TCMDC-137848, -142296 and -142051). 
Supplemental Data S2: Positive controls with Fluo-4 redistribution

\begin{tabular}{|c|c|c|c|c|c|c|c|c|c|c|c|c|c|c|c|c|c|c|c|c|c|}
\hline \multirow{2}{*}{ TCAMs code } & \multirow{2}{*}{$\begin{array}{l}\text { DV Disruption } \pm \\
\text { SEM (\%) }\end{array}$} & \multirow{2}{*}{$\begin{array}{c}\text { Mitochondrial } \\
\text { Intactness } \pm \text { SEM (\%) }\end{array}$} & \multirow{2}{*}{$\begin{array}{l}\text { DNA Intactness } \pm \\
\text { SEM (\%) }\end{array}$} & \multirow{2}{*}{ Cluster } & \multirow{2}{*}{$\begin{array}{c}\text { cytotox } \\
\text { HepG2(HM) }\end{array}$} & \multicolumn{3}{|c|}{ 1st assessment of cross-resistance in W2 } & \multicolumn{7}{|c|}{ 2nd assessment of cross-resistance, mini-panel } & \multirow{2}{*}{ 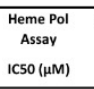 } & \multicolumn{3}{|c|}{$\begin{array}{l}\text { Heme Pol Assay, \%of activity with respect to } \\
\text { control at different concentrations }\end{array}$} & \multirow{2}{*}{ GC IC50 ( $\mu \mathrm{M})$} & \multirow{2}{*}{ PRR } \\
\hline & & & & & & 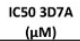 & $1550 \mathrm{~W} 2(\mu \mathrm{MM})$ & Ratio W2/307 & $\begin{array}{c}1650307 \mathrm{~A} \\
(\mu \mathrm{M})\end{array}$ & $1 \mathrm{CSO} \mathrm{k1} 1(\mu \mathrm{M})$ & $\begin{array}{l}\text { IC50 тM90с2B } \\
(\mu \mathrm{M})\end{array}$ & $\begin{array}{c}\text { ICSO V1/s } \\
(\mu M)\end{array}$ & Ratio K1/307 & 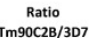 & $\begin{array}{c}\text { Ratio } \\
\text { V1 } 5 / 307\end{array}$ & & $100 \mu \mathrm{M}$ & $50 \mu \mathrm{M}$ & $10 \mu \mathrm{m}$ & & \\
\hline 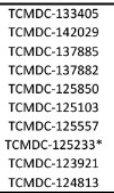 & 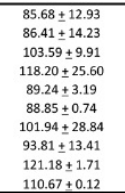 & $\begin{array}{l}16.54+4.21 \\
7.25+0.53 \\
16.68+0.31 \\
39.14+7.95 \\
21.36+4.37 \\
26.43+7.72 \\
19.74+5.68 \\
19.02+2.81 \\
23.16+4.76 \\
21.13+1.79 \\
21.13\end{array}$ & 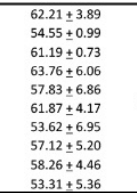 & Chloroquine-like & $23.83 \pm 1.00$ & $0.08 \pm 0.01$ & $2.89 \pm 0.00$ & 37.48 & $0.04 \pm 0.01$ & $1.75 \pm 0.59$ & $0.96 \pm 0.12$ & $2.98 \pm 0.34$ & 46.60 & 25.50 & 79.30 & & $100.81 \pm 0.36$ & $100.23 \pm 0.42$ & $38.98 \pm 6.38$ & $>10$ & FAST \\
\hline TCMDC-142276 & $118.24 \pm 2.87$ & $17.62+7.34$ & $54.32 \pm 5.23$ & $\begin{array}{l}\text { Amodiaquine- } \\
\text { like }\end{array}$ & & $0.03 \pm 0.00$ & $0.07 \pm 0.00$ & 2.33 & & & & & & & & & $100.38 \pm 0.08$ & $100.12+0.40$ & $65.92 \leftleftarrows 16.19$ & & \\
\hline 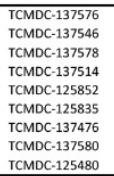 & 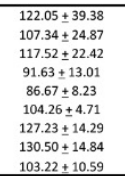 & $\begin{array}{l}10.14+1.45 \\
4.55+0.89 \\
6.08+0.37 \\
6.77+0.36 \\
53.33+7.80 \\
8.30+0.03 \\
6.85+1.92 \\
8.89+0.17 \\
11.26+6.38 \\
\end{array}$ & $\begin{array}{l}58.15 \pm 5.91 \\
56.564 .48 \\
44.79 \pm 1.43 \\
53.0 \pm 0.99 \\
60.31 \pm 0.44 \\
60.0 \pm 2.79 \\
52.3 \pm \pm 4.96 \\
53.31 \pm 4.56 \\
61.10 \pm 6.02 \\
\end{array}$ & Acridine-like & $\begin{array}{l}4.74 \pm 0.18 \\
4.87 \pm 2.84 \\
14.57 \pm 1.99\end{array}$ & $\begin{array}{l}0.12 \pm 0.02 \\
0.08 \pm 0.01 \\
0.10 \pm 0.00\end{array}$ & $\begin{array}{l}0.73 \pm 0.02 \\
0.09 \pm 0.01 \\
1.28 \pm 0.30\end{array}$ & $\begin{array}{l}>6.7 \\
>11.6 \\
6.36 \\
1.19 \\
12.93\end{array}$ & $\begin{array}{l}0.04 \pm 0.03 \\
0.06+0.01\end{array}$ & $\begin{array}{l}0.31 \pm 0.06 \\
0.09+0.01\end{array}$ & $\begin{array}{l}0.22 \pm 0.04 \\
0.11+0.04\end{array}$ & $\begin{array}{l}0.36 \pm 0.12 \\
0.20+0.09\end{array}$ & $\begin{array}{l}7.19 \\
1.50\end{array}$ & $\begin{array}{l}4.93 \\
1.77\end{array}$ & $\begin{array}{l}8.28 \\
3.21\end{array}$ & $\begin{array}{l}9.81+2.36 \\
14.84+0.70\end{array}$ & $\begin{array}{l}100.07 \pm 0.41 \\
81.10 \pm 26.45\end{array}$ & $\begin{array}{l}98.18+0.68 \\
86.83 \pm 2.28\end{array}$ & $\begin{array}{l}9.05 \pm 10.31 \\
-4.57 \pm 4.84\end{array}$ & $\begin{array}{l}1.99 \pm 0.07 \\
5.17 \pm 0.04\end{array}$ & $\begin{array}{l}\text { FAST } \\
\text { FAST }\end{array}$ \\
\hline
\end{tabular}

* TCMDC-125233 selected for full profiling as a CQ-like compound representative. 
Supplemental Data S3: Positive controls with no Fluo-4 redistribution

\begin{tabular}{|c|c|c|c|}
\hline TCAMs code & $\begin{array}{c}\mathrm{XC50}(\mu \mathrm{M}) \\
\text { screening Pf } \\
\text { data }\end{array}$ & Cluster & DV-disruption (\%) \\
\hline TCMDC-133399 & 0.226 & & 53.84583857 \\
\hline TCMDC-133398 & 0.697 & & 40.90819745 \\
\hline TCMDC-137733 & 0.482 & & 67.39818447 \\
\hline TCMDC-123790 & 0.532 & & 37.30798732 \\
\hline TCMDC-142277 & 0.025 & & 59.50908208 \\
\hline TCMDC-142279 & 0.017 & & 53.74265243 \\
\hline TCMDC-142247 & 0.488 & & 42.06372691 \\
\hline TCMDC-133571 & 0.315 & & 36.23677817 \\
\hline TCMDC-133574 & 0.909 & & 55.72125528 \\
\hline TCMDC-133584 & 0.785 & & 32.82307612 \\
\hline TCMDC-125876 & 0.200 & & 68.49415132 \\
\hline TCMDC- 137588 & 0.067 & & 53.21531367 \\
\hline TCMDC-123987 & 0.762 & & 67.30896086 \\
\hline & & Chloroquine-like & \\
\hline TCMDC-123988 & 0.178 & & 60.83590279 \\
\hline TCMDC-133668 & 0.484 & & 59.35402718 \\
\hline TCMDC-133568 & 0.178 & & 62.422975 \\
\hline TCMDC-125506 & 0.339 & & 42.81186638 \\
\hline TCMDC-133406 & 0.120 & & 54.14827478 \\
\hline TCMDC-133572 & 0.357 & & 60.79352076 \\
\hline TCMDC-133662 & 0.298 & & 19.51929843 \\
\hline TCMDC-133666 & 0.248 & & 37.13045941 \\
\hline TCMDC-134027 & 0.753 & & 16.41197895 \\
\hline TCMDC-125106 & 0.266 & & 27.52410177 \\
\hline TCMDC-125231 & 0.315 & & 50.69459303 \\
\hline TCMDC- 125232 & 0.655 & & 32.63234749 \\
\hline TCMDC- 125528 & 0.744 & & 29.49360114 \\
\hline TCMDC-141999 & 0,715 & \multirow{4}{*}{$\begin{array}{l}\text { Acridine- and } \\
\text { chloroquine-like }\end{array}$} & 63.12288164 \\
\hline TCMDC- 137460 & 0.175 & & 44.73524894 \\
\hline TCMDC-138329 & 0.787 & & 55.72263085 \\
\hline TCMDC-124272 & 0.800 & & 22.21076418 \\
\hline TCMDC-124265 & 0.878 & \multirow{9}{*}{ Acridine-like } & 54.49164141 \\
\hline TCMDC-138009 & 0.117 & & 33.93784133 \\
\hline TCMDC-137547 & 0.233 & & 57.46278735 \\
\hline TCMDC-138018 & 0.587 & & 61.11376842 \\
\hline & & & \\
\hline TCMDC-137569 & 0.167 & & 62.45551521 \\
\hline TCMDC-137572 & 0.512 & & 58.2955691 \\
\hline TCMDC-125004 & 0.802 & & 46.78568462 \\
\hline TCMDC-138079 & 0.266 & & 63.78834946 \\
\hline
\end{tabular}

*TCMDC-123988 is chloroquine, but it did not exhibit calcium disruption during the screen. This may be due to variability of the assay, impurity of the sample or diluent effects. The CQ used as positive controls on all assay days was diluted in PBS, aliquoted and frozen whereas the CQ dispensed in the drug plate was dissolved in DMSO and may be subjected to freeze thaw effects. 
Supplemental Data S4: Negative controls

\begin{tabular}{lccc}
\hline TCAMs code & Cluster & DV-disruption (\%) & Validation assays \\
\hline Artemisinine & NIL & 32.86127642 & Not performed \\
\hline Mefloquine & NIL & 41.27 & Not performed \\
\hline & & & $\begin{array}{c}\text { Shown to be a deviant (intact } \\
\text { mitochondria and DNA); DV not } \\
\text { disrupted when used Fura-red } \\
\text { TCMDC-141416 }\end{array}$ \\
& & 88.5363611 & $\begin{array}{c}\text { assay to measure Ca }{ }^{2+} \text { in parasite } \\
\text { cytosol after drug treatment }\end{array}$ \\
TCMDC-141335 & & & Not performed \\
TCMDC-141330 & Quinine-like & & Not performed \\
TCMDC-141415 & & 40.26740999 & Not performed \\
TCMDC-141417 & & 45.31809085 & Not performed \\
TCMDC-137467 & & 48.4377379 & Not performed \\
TCMDC-124047 & & 49.03102613 & Not performed \\
TCMDC-141333 & 36.48452138 & Not performed \\
TCMDC-131239 & & 61.98206587 & Not performed \\
\hline
\end{tabular}




\section{Supplemental Data S5}

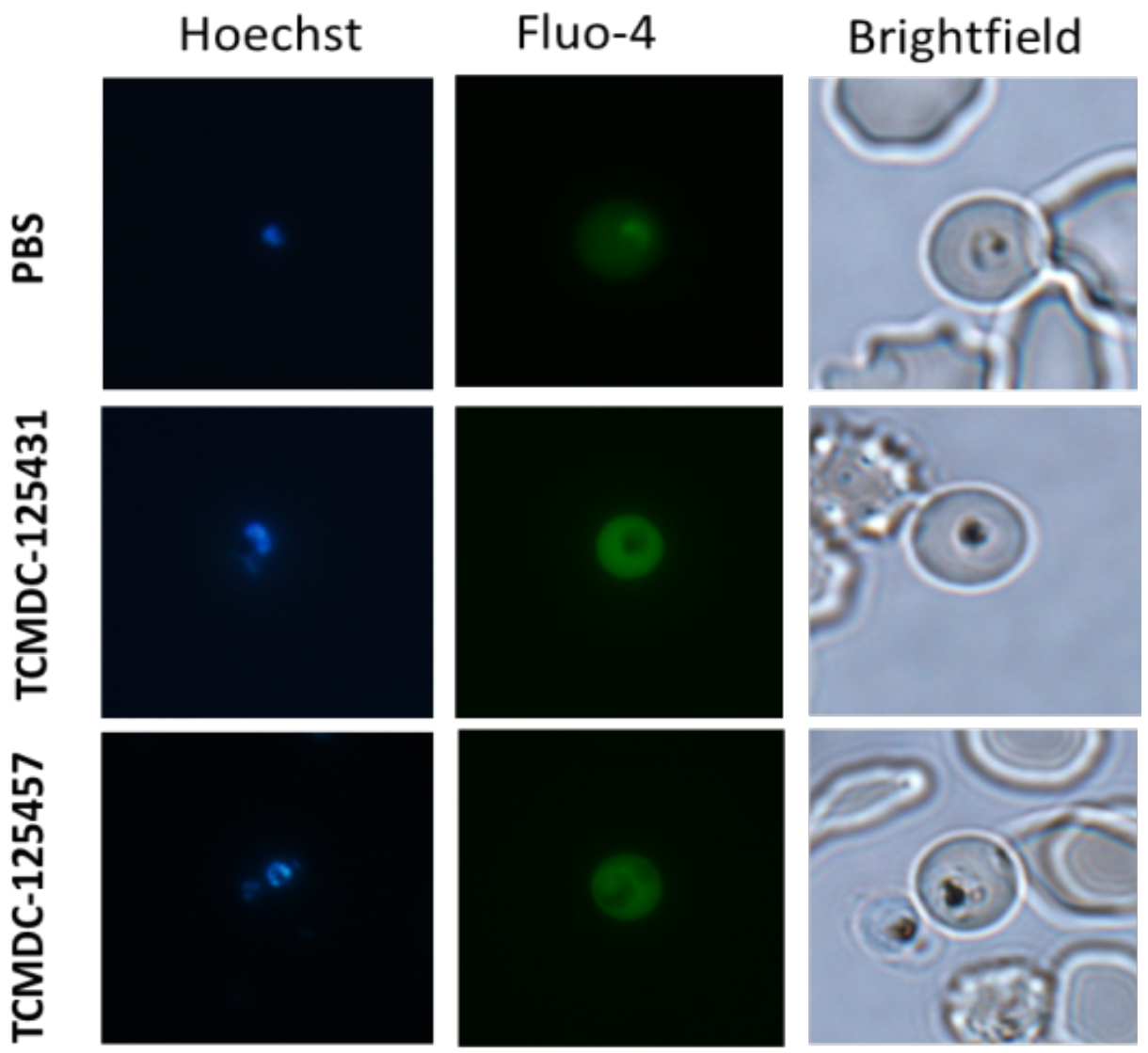

Fluorescence microscopy images of compound-treated parasites indicative of altered calcium distribution (Green indicates Flou-4 AM signal for calcium and Blue indicates Hoechst 33342 for parasite DNA), against control (PBS) treated parasites. 
Supp Data S6: Drug resistant pattern of $P$. falciparum strains

\begin{tabular}{|c|c|c|c|c|c|c|c|c|c|}
\hline \multirow[t]{2}{*}{ Strains } & \multirow[t]{2}{*}{ MR4 \# } & \multicolumn{6}{|c|}{ Drug resistance* } & \multirow[t]{2}{*}{ Source } & \multirow[t]{2}{*}{ Reference } \\
\hline & & CQ & PYR & CYC & SUL & ATO & ART & & \\
\hline 3D7 & MRA-102 & $\mathrm{s}$ & $\mathrm{s}$ & $\mathrm{s}$ & $\mathrm{R}$ & & & $\begin{array}{l}\text { Clone from the NF4 strain; } \\
\text { Netherlands isolate }\end{array}$ & Walliker (1987) \\
\hline 3D7A & MRA-151 & $S$ & $\mathrm{~s}$ & $\mathrm{~s}$ & & & & $\begin{array}{l}\text { Subclone from 3D7 } \\
\text { standard for the } P \text {. } \\
\text { falciparum genome } \\
\text { sequencing project }\end{array}$ & \\
\hline PM2GT & MRA-805 & & & & & & & $\begin{array}{l}\text { Parental strain: 3D7 } \\
\text { plasmepsin II-GFP fusion }\end{array}$ & Klemba (2004) \\
\hline W2 & MRA-157 & $\mathrm{R}$ & $\mathrm{R}$ & $\mathrm{R}$ & $\mathrm{R}$ & & & $\begin{array}{l}\text { Indochina III/CDC isolate } \\
\text { from Lao }\end{array}$ & Oduola (1988) \\
\hline K1 & MRA-159 & $R$ & $R$ & $R$ & $R$ & & & Thailand isolate & Thaithong (1981) \\
\hline $\mathrm{V} 1 / \mathrm{s}$ & MRA-176 & $\mathrm{R}$ & $\mathrm{R}$ & $\mathrm{R}$ & $\bar{R}$ & & & $\begin{array}{l}\text { in vitro culture-adapted } \\
\text { clone of the } \mathrm{V} 1 \text { strain, } \\
\text { origin Vietnam }\end{array}$ & Rosario (1981) \\
\hline TM90C2B & $N / A$ & $\mathrm{R}$ & $\mathrm{R}$ & & & $\mathrm{R}$ & & Thailand isolate & $\begin{array}{l}\text { Looareesuwan } \\
\text { (1996) }\end{array}$ \\
\hline $\begin{array}{l}\text { IPC 5202 } \\
\text { (Cam3.IR539T) }\end{array}$ & MRA-1240 & & & & & & $\mathrm{R}$ & Cambodian isolate & Straimer (2015) \\
\hline
\end{tabular}

CQ: chloroquine; PYR: pyrimethamine; CYC: Cycloguanil; SUL: sulfadoxin; ATO: atovaquone; ART: artemisinin

*S: sensitive, $\mathrm{R}$ : resistant, as reported in the literature and MR4 resource.

\section{REFERENCES}

Klemba M, Beatty W, Gluzman I, Goldberg DE, Trafficking of plasmepsin II to the food vacuole of the malaria parasite Plasmodium falciparum, J Cell Bio 2004, 164(1):47-56.

Looareesuwan S, Viravan C, Webster HK, Kyle DE, Hutchinson DB, Canfield CJ: Clinical studies of atovaquone, alone or in combination with other antimalarial drugs, for treatment of acute uncomplicated malaria in Thailand. AmJTrop Med Hyg 1996, 54:62.

Oduola, A. M. J., N. F. Weatherly, J. H. Bowdre, and R. E. Desjardins. Plasmodium falciparum: cloning by single erythrocyte manipulation and heterogeneity in vitro. Exp. Parasitol. 1988; 66:86-95.

Rosario V: Cloning of naturally occurring mixed infections of malaria parasites. Science 1981, 212:1037-103.

Straimer, J., et al. K13-Propeller Mutations Confer Artemisinin Resistance in Plasmodium falciparum Clinical Isolates. Science 2015; 347: 428- 431.

Thaithong S, Beale GH: Resistance of ten Thai isolates of Plasmodium falciparum to chloroquine and pyrimethamine by in vitro tests. Trans $R$ Soc Trop Med Hyg 1981, 75:271273.

Walliker D, Quakyi I.A, Wellems T.E, McCutchan T.F, Szarfman A, London W.T, Corcoran L.M, Burkot T.R, Carter R. Genetic analysis of the human malaria parasite Plasmodium falciparum. Science 1987, 236: 1661- 1666. 